\title{
Pre-conditioning and post-conditioning in myocardial infarction.
}

\author{
Liviu Macovei ${ }^{1,2 *}$, Larisa Anghe $1^{1,2}$ \\ ${ }^{1}$ Grigore T. Popa University of Medicine and Pharmacy, Romania \\ ${ }^{2}$ George I.M. Georgescu Institute of Cardiovascular Diseases, Romania
}

Accepted on October 06, 2017

Despite the special attention in the context of myocardial reperfusion in acute myocardial infarction (thrombolysis or primary angioplasty), the mortality of these patients continues to remain high. New ways of therapeutic intervention are therefore needed to improve the quality of life of these patients and also to reduce the size of myocardial necrosis. This is one of the new targets in the management of patients with acute myocardial infarction [1].

Myocardial reperfusion injury post primary angioplasty in acute myocardial infarction includes myocardial sideration, microvascular sideration, reperfusion arrhythmias and lethal reperfusion with myocardial necrosis.

Ischemic pre- and post-conditioning are two endogenous cardioprotective mechanisms that cause a significant increase in the ability of the heart to cope with ischemic injury.

Ischemic preconditioning represents the adaptation of the myocardium to hypoperfusion caused by recurrent ischemic episodes, with clinical benefit of decreasing the infarct size and reducing the severity of contractile dysfunction.

The formal description of ischemic preconditioning as an experimental phenomenon capable of significantly limiting the size of the infarction was made by Murry et al. [2]. They demonstrated that in the case of an anesthetized dog, 5-minute occlusion of the anterior descending artery followed by 5 minutes of myocardial reperfusion before the 40-minute occlusion of the same artery, resulted in a significant reduction of the infarct size. This cardioprotective effect of ischemic preconditioning was independent of changes in transmural myocardial circulation, so Murry et al. claimed that it is secondary to rapid metabolic adaptation of the ischemic myocardium. The wide reproducibility of this phenomenon through various preconditioning protocols led to the recognition of ischemic preconditioning as a "gold standard" for cardioprotection [2].

There have been described several molecular mechanisms that are involved in ischemic preconditioning:

Protein kinases: They are phosphorylated, translocated or have increased activity in the pre-conditioned myocardium [3] and the transient preischemic activation of $\mathrm{G}$ protein receptors through synthetic ligands or exogenous carcinoids, provides protection ("pharmacological preconditioning"), which is several times similar to ischemic preconditioning [4].
ATP-dependent $\mathrm{K}+$ channels: They open during ischaemia, hypoxia or metabolic inhibition, thus facilitating an increase in $\mathrm{K}$ influx and shortening the duration of action potential, thus having an aritmogenic effect $[5,6]$.

Signaling pathways: Induces increased synthesis of nitric oxide, activation of soluble guanylil cyclase, cyclic GMP accumulation and activation of cyclic GMP dependent protein kinases 6 .

\section{Ischemic post-conditioning}

Myocardial reperfusion initiates a cascade of events in the first few minutes of restoring blood flow and induces myocardial injury shortly.

Reducing the size of the infarct via ischemic postconditioning varies with the duration of ischaemia. Experimental studies show that with the prolongation of the duration of myocardial ischaemia, the potential to reduce the size of the infarction is limited, possibly due to the fact that the extent of ischemic injury is more severe [7]. By reducing the duration of ischaemia, reperfusion interventions can cause a significant reduction in the size of the infarct $[5,8]$.

The beneficial effect, both on short, but especially on longterm, of the post-conditioning protocols on left ventricular function had been observed in many studies $[9,10]$ significantly reducing the incidence of reperfusion arrhythmias.

The degree of protection provided by ischemic post-conditioning depends on the duration of the ischaemia and the post-conditioning protocol used. So far, there is insufficient data to study how different factors such as classical cardiovascular risk factors, medication, gender or age influence the effectiveness of post-conditioning [11].

Factors that have an important role in ischemic postconditioning are:

Adenosine: There are studies that support the role of A2 receptor agonists on limiting infarct size in different species [1,7].

Opioids: Activation of endogenous opioid receptors contributes to ischemic post-conditioning in rodents; also, the exogenous administration of opioid receptor agonists would favor ischemic post-conditioning [8].

Bradykinin: Appears to be involved in ischemic postconditioning, but the mechanism of action is unknown $[1,9]$. 
Calcium and calpain: The accumulation of calcium ions at the cytosolic and mitochondrial level after a period of hypoxia followed by reoxygenation was reduced by cardiomyocyte ischemia post-conditioning; it has also been observed that transient acidosis during reperfusion reduces calpain-mediated proteolysis and reperfusion injuries $[1,10]$.

In a case-control clinical trial, patients with a first STEMI benefited from ischemic post-conditioning after primary angioplasty, by swelling a balloon over the stent in 4 cycles of one minute. In these patients, the size of myocardial necrosis was significantly lower, both early and late after STEMI [11].

Remote preconditioning expresses the cardioprotection resulting from the activation of reperfusion injury mechanisms in a territory other than myocardial. Thus, by repeated swelling and deflagration of a upper limb tension cuff ( 3 cycles of $5 \mathrm{~min}$ ), reperfusion injury mechanisms are activated at this level (neutrophil activation and blocking of mitochondria due to increased calcium influx), with the limitation of the adverse effects at the myocardial level after primary angioplasty [1].

Acute myocardial infarction is considered a standard in emergency cardiology, and the advances made over the last decade in terms of discovering the molecular mechanisms involved in atherothrombosis are remarkable. These researches have made it possible to identify vulnerable plaques, find ways to prevent destabilization of the atherosclerotic plaque and once acute coronary syndrome occur, limiting the consequences of coronary thromboembolism.

What has not yet been achieved is limiting the reperfusion injury that amplifies cellular necrosis due to the ischemic thrombotic process. Ischemic conditioning, especially by post-conditioning and remote conditioning, is trying to do this, but so far favorable results have been obtained only sporadically.

It is necessary to define an optimal protection protocol for certain duration, degree of myocardial ischaemia and a certain size of the myocardial area at risk, but also depending on the extension of the collateral circulation.

\section{References}

1. Macovei L, Presura RM, Arsenescu Georgescu C. Systemic or local thrombolysis in high-risk pulmonary embolism. Cardiol J. 2015;22:467-74.
2. Murry CE, Jennings RB, Reimer KA. Preconditioning with ischemia: a delay of lethal cell injury in ischemic myocardium. Circulation. 1986;74:1124-36.

3. Budas GR, Churchill EN, Mochly-Rosen D. Cardioprotective mechanisms of PKC isozyme-selective activators and inhibitors in the treatment of ischemia reperfusion injury. Pharmacol Res. 2007;55:523-36.

4. Ovize M, Baxter GF, Di Lisa F, et al. Post-conditioning and protection from reperfusion injury: Where do we stand? Cardiovasc Res. 2010;87(3):406-23.

5. Ferdinandy P, Schultz R, Baxter G. Interaction of cardiovascular risk factors with myocardial ischemia/ reperfusion injury, pre-conditioning and postconditioning. Pharmacol Rev. 2007;59:418-58.

6. Brennan JP, Southworth R, Medina RA, et al. Mitochondrial uncoupling, with low concentration FCCP, induces ROS dependent cardioprotection independent of KATP channel activation. Cardiovasc Res. 2006; 72:313-21.

7. Piper HM, Garcia-Dorado D, Ovize M. A fresh look at reperfusion injury. Cardiovasc Res. 1998;38:291-300.

8. Wang G, Zhang S, Joggerst SJ, et al. Effects of the number and interval of balloon inflations during primary PCI on the extent of myocardial injury in patients with STEMI: Does post-conditioning exist in real-world practice? J Invasiv Cardiol. 2009;21:451-5.

9. Mewton N, Croisille P, Gahide G, et al. Effect of cyclosporine on left ventricular remodeling after reperfused myocardial infarction. J Am Coll Cardiol. 2010;55:1200-5.

10. Garcia-Dorado D, Ruiz-Meana M, Piper HM. Lethal reperfusion injury in acute myocardial infarction: Facts and unresolved issues. Cardiovasc Res. 2009;83:165-8.

11. Sivaraman V, Mudalgiri NR, Di SC, et al. Postconditioning protects human atrial muscle through the activation of the RISK pathway. Basic Res Cardiol. 2007; 102:453-9.

\author{
*Correspondence to: \\ Liviu Macovei \\ Senior Physician \\ "George I.M. Georgescu" Institute of Cardiovascular \\ Diseases \\ Iasi \\ Romania \\ E-mail: liviughemacobei@yahoo.com
}

